\title{
Editorial
}

\section{Modeling and Control of Complex Dynamic Systems 2014}

\author{
Zhiwei Gao, ${ }^{1}$ De-Xing Kong, ${ }^{2}$ and Michael Z. Q. Chen ${ }^{3}$ \\ ${ }^{1}$ Faculty of Engineering and Environment, Northumbria University, Newcastle upon Tyne NE1 8ST, UK \\ ${ }^{2}$ Department of Mathematics, Zhejiang University, Hangzhou 310027, China \\ ${ }^{3}$ Department of Mechanical Engineering, The University of Hong Kong, Hong Kong Island, Hong Kong \\ Correspondence should be addressed to Zhiwei Gao; zhiwei.gao@northumbria.ac.uk
}

Received 31 December 2014; Accepted 31 December 2014

Copyright (C) 2015 Zhiwei Gao et al. This is an open access article distributed under the Creative Commons Attribution License, which permits unrestricted use, distribution, and reproduction in any medium, provided the original work is properly cited.

\section{Introduction}

Nowadays, practical dynamic systems have become more and more complex, which may be characterized by a model subjected to environmental uncertainties, communication time delays, stochastic perturbations, hybrid dynamics, distributed dynamics, chaotic dynamics, collective behaviours, and so forth $[1,2]$. As a result, modeling and control of a complex system either from the viewpoint of mathematical theoretical methods or the view of real-time application point have been an interesting but challenging research topic. This is the third-year special issue from which one may see some recent research progresses in this research field. The special issue received 63 submissions, and 6 papers are selected after a peer-review procedure, indicating an acceptance rate of less than $10 \%$.

\section{Modeling and Analysis of Complex Dynamic Systems}

Modeling is a fundamental work which is always a starting point for control, optimisation, and implementation. In this special issue, there are three papers focused on the investigation of the modeling issues.

In the work entitled "Mathematical Modeling of Transient Responses in a Large Scale Multiconductor System" contributed by C. Sheng and X. Zhang, a novel reducedorder macromodeling algorithm is proposed for calculating the lightning transient responses in the lightning protection systems of the structures. In this study, the block Arnoldi algorithm is utilized for reducing the matrix dimension. The computed lightning transient responses in a lightning protection system are demonstrated to conform reasonably to those from experimental measurements, which confirm the validity of the proposed model.

Nonlinearity is one of the distinguished properties of a complex dynamic system, which has brought challenges in systems modeling. In the paper "Nonlinear Torsional Vibration Dynamics Behaviors of Rolling Mill's Multi-DOF Main Drive System under Parametric Excitation," authored by D. Han et al., the nonlinear torsional vibration dynamical model of the multi-DOF rolling mill's main drive system under joint angle parametric excitation is built. In the study, the multiscale method is used to evaluate the nonlinear dynamic behaviour of the torsional system by analysing the amplitudefrequency characteristic equation and amplitude-frequency response equation. The employed modeling method is validated by the numerical example of the 1780 rolling-mill main drive system.

A radio frequency (RF) power amplifier is a typical nonlinear system. Even when the transistor is operating in a quasilinear region, driven by a small variant input signal, the output signal is still nonlinear due to the physics of the transistor. In the paper entitled "Evolution of Black-Box Models based on Volterra Series," contributed by D. D. Silveira et al., a modern classification of the behavioural models of the RF power amplifiers is discussed. An evolution of the behavioural models is reviewed from the basic baseband power series to the recent parallel multirate pruned Volterra series models. Some comments on the future trends in power amplifiers behavioural modeling are also provided.

Another fundamental work is the existence analysis of the solutions to dynamic equations that describes a practical 
process. In the paper entitled "On the Existence of Global Weak Solutions for a Weakly Dissipative Hyperelastic Rod Wave Equation," contributed by $\mathrm{H}$. Yan et al., the existence of the global weak solutions to the weakly dissipative hyperelastic rod wave equation is investigated. By using the limit of the viscous approximations to the equation and the estimates derived from the equation itself, the existence of the global weak solution is addressed with rigorous mathematical proofs.

\section{Application Research of Complex Dynamic Systems}

As the development and application of clean energy such as wind power, solar panel, marine and wave energy, electric vehicles, CHP, and CCHP engines, the future power network is becoming distributed and the power flow is turning into bidirectional flow. As a result, there is an increasing attention on the research of the energy storage in the future power network.

In the paper "A New Pricing Scheme for Controlling Energy Storage Devices in Future Smart Grid," contributed by J. Zhu et al., a universal pricing scheme for controlling energy storage devices in future smart grid is proposed, which is designed to efficiently reduce the value of any convex objective function defined by the grid operators. Simulation results have demonstrated the stability and profitability of the present pricing scheme. The proposed pricing scheme can be applied to control the behaviour of the energy storage devices installed for the integration of the current intermittent renewable energy and would have much broader applications in the future.

As the development of the wireless sensors and wireless sensor network, the modern automation industry has benefited from using wireless sensor based control and monitoring techniques. It is noticed that robustness, scalability, power consumption, memory resources, and computation ability are the main constraints of wireless sensor networks, which have limited the wireless sensor based control and monitoring performance. In order to overcome the constraints of wireless sensor networks, gossip algorithm has attracted research attention due to its distributed computation ability.

In the work "Nonlinear Gossip Algorithms for Wireless Sensor Networks," authored by C. Shi et al., nonlinear gossip algorithms for wireless sensor networks are investigated. The proposed gossip algorithm can converge to the average of the initial values with probability one. In addition, the proposed algorithm is a general version, as the traditional linear gossip algorithm can be viewed as a special case of the present nonlinear gossip algorithm. The paper also points out that how to determine and accelerate the convergence rate of the nonlinear gossip algorithms is an issue needed to be further investigated.

\section{Conclusion}

Complex dynamic system is a rather broad research filed, whose researches are motivated by a variety of practical engineering systems or social, economic, and ecological concerns.
Modeling, control, and optimisation are major research issues for complex dynamic systems. We anticipate that this special issue would provide a bit of light to the readers on what and how the research topic has been developed recently.

\section{Acknowledgments}

Sincere thanks are addressed to all the authors who responded to the call of the special issue. The guest editor team is deeply indebted to the volunteer contributions from the reviewers.

\section{Zhiwei Gao De-Xing Kong Michael Z. Q. Chen}

\section{References}

[1] Z. Gao, D. Kong, and C. Gao, "Modeling and control of complex dynamic systems: applied mathematical aspects," Journal of Applied Mathematics, vol. 2012, Article ID 869792, 5 pages, 2012.

[2] Z. Gao, D. Kong, C. Gao, and M. Chen, "Modeling and control of complex dynamic systems 2013," Journal of Applied Mathematics, vol. 2013, Article ID 151372, 3 pages, 2013. 


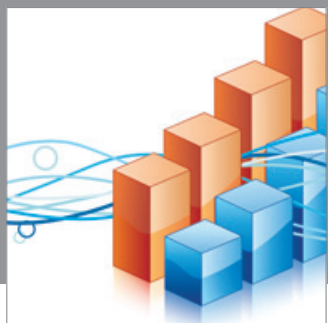

Advances in

Operations Research

mansans

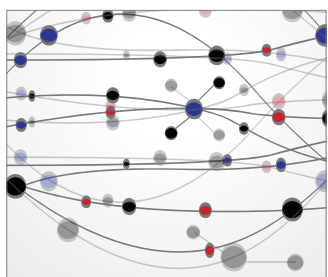

The Scientific World Journal
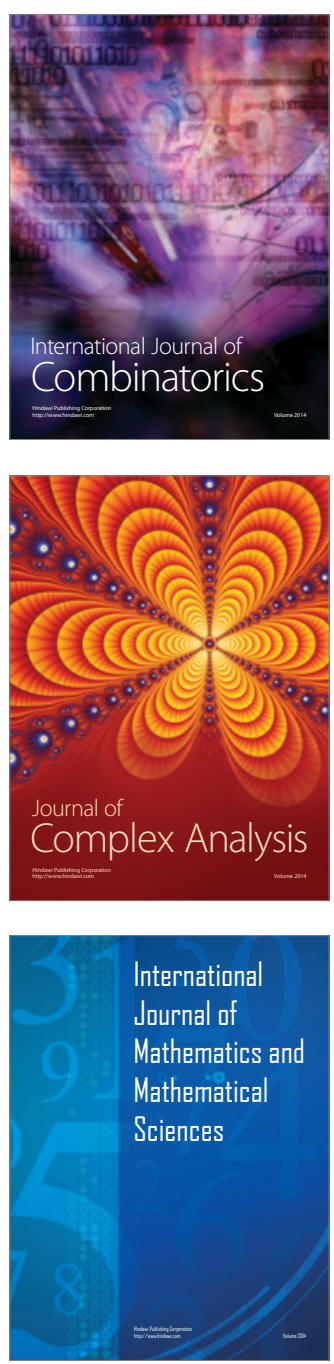
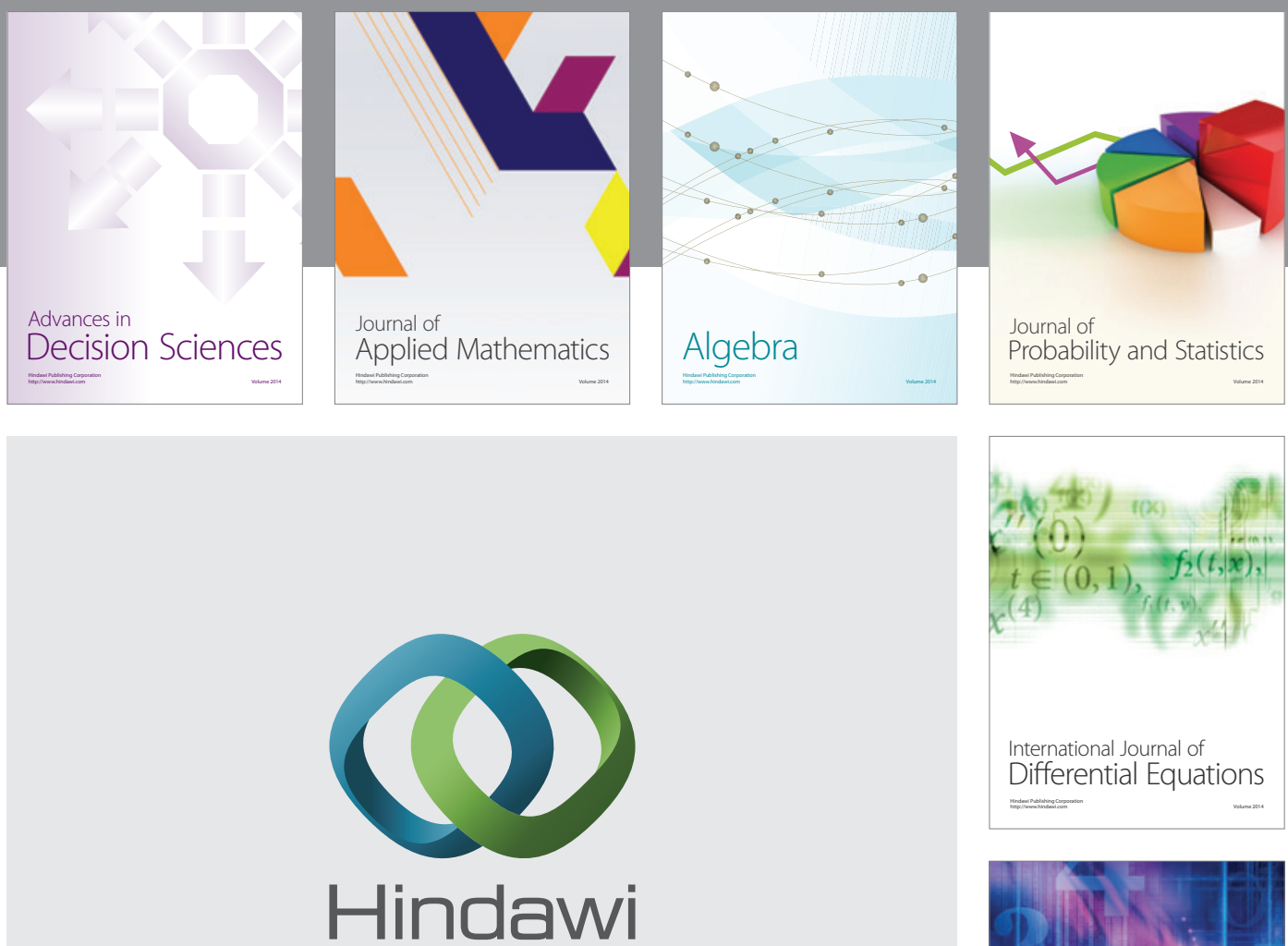

Submit your manuscripts at http://www.hindawi.com
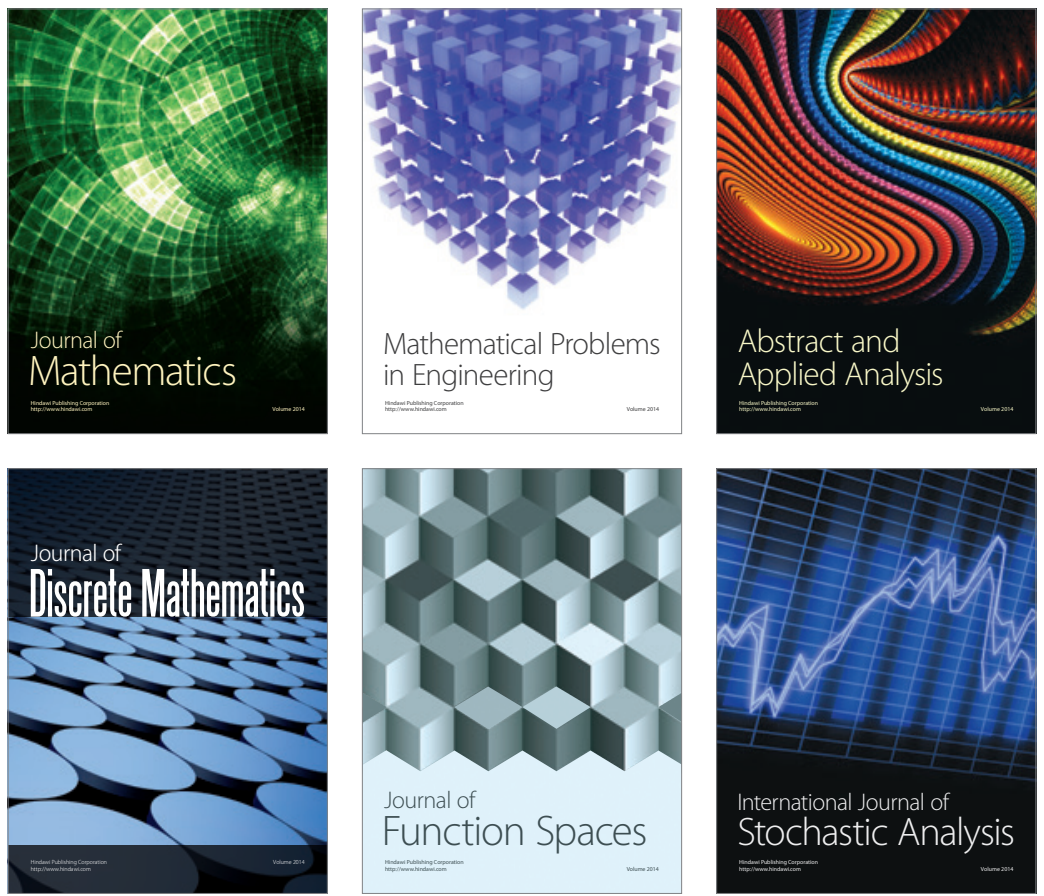

Journal of

Function Spaces

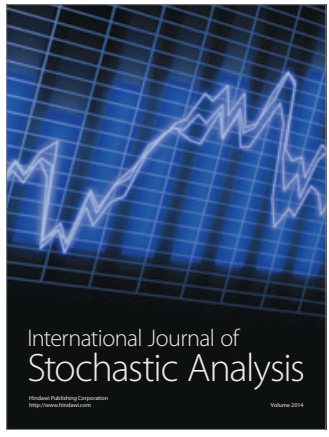

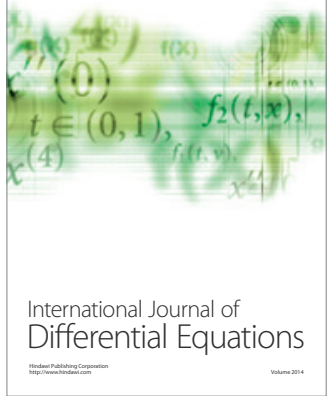
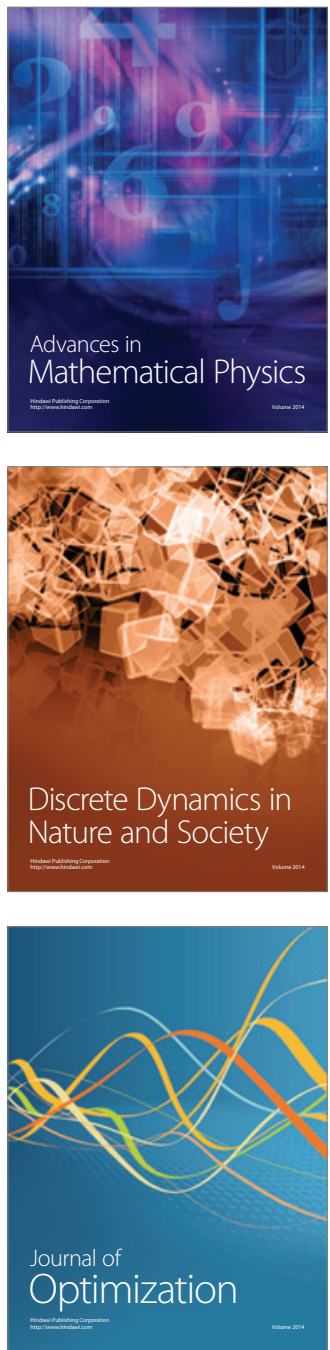